\title{
Health-related quality of life and utility
}

(a) CrossMark values associated to hypoglycemia in patients with type 1 diabetes mellitus treated in the Brazilian Public Health System: a multicenter study

Luciana Bahia ${ }^{1,6^{*}}$, Rosane Kupfer², Denise Momesso², Debora A. P. Cabral ${ }^{2}$, Balduino Tschiedel ${ }^{3}$, Marcia Puñales ${ }^{3}$, Suzana Lavigne ${ }^{3}$, Cristina F. S. Façanha ${ }^{4}$, Adriana C. Forti ${ }^{4}$, Angela D. N. Mendes ${ }^{4}$ and Bernardo R. Tura ${ }^{5}$

\begin{abstract}
Background: Hypoglycemia is a critical and limiting factor of a good metabolic control and can adversely affect the quality of life of diabetic patients. The aim of the study was to evaluate the health-related quality of life and calculate utilities values associated with hypoglycemia in patients with type 1 diabetes mellitus (T1DM).

Methods: A multicenter, cross-sectional and observational study with T1DM patients from reference centers of the Brazilian public health system was conducted in three cities. Demographic and clinical data were collected, besides details on the frequency and severity of hypoglycemia. Health-related quality of life was assessed using EQ-5D instrument and utility values generated.

Results: 221 patients (107 women, 114 men), aged $29.8 \pm 11.6$ and disease duration of $14.2 \pm 9.1$ years were included. Most patients $(n=214,96.8 \%)$ reported at least one symptomatic hypoglycemia in the last three months, $68 \%(n=150)$ reported nocturnal episodes and 34.8\% $(n=77)$ reported severe episodes. High frequency (daily or weekly) was observed in 38.6 and $26 \%$ of those reporting nocturnal or severe hypoglycemia, respectively. The median visual analog scale was 70 [60-85] for all patients, with differences between those with and without severe hypoglycemia (70 [60-80] vs 80 [61-90]; $p=0.006)$ and those with high and low frequency (62.5 [50-72.25] vs 70 [60-80]; $p=0.007)$. The median utility values was $0.801[0.756-1.000]$ for all patients, with difference between those with high and low frequency of severe episodes (0.737 [0.628-1.000] vs 0.801 [0.756-1.000]; $p=0.02$ ).
\end{abstract}

Conclusions: This study shows the high frequency of hypoglycemia in a sample of T1DM patients treated in three reference centers of the Brazilian public health system and the impact of severe episodes on health-related quality of life. Utility values were generated and can be used in economic analysis for treatments that could decrease hypoglycemia and consequently improve quality of life.

Keywords: Quality of life, Utility, Hypoglycemia, Diabetes

\section{Background}

Hypoglycemia is a common event in the lives of patients with type 1 diabetes mellitus (T1DM) and carries high

\footnotetext{
*Correspondence: Iucianabahia@gmail.com

${ }^{6}$ Visconde de Pirajá 547/501 Ipanema, Rio de Janeiro 22410-003, Brazil

Full list of author information is available at the end of the article
}

morbidity. The goal of diabetes treatment is to achieve euglycemia without episodes of hypoglycemia, but the frequent occurrence of hypoglycemia is a critical and limiting factor of a good metabolic control in the short and long-term [1]. The episodes may be asymptomatic and go undetected, quickly leading to alterations of consciousness and coma, or accompanied by extremely 
uncomfortable and disturbing symptoms associated with decreased quality of life. Often patients with diabetes fear more the severe hypoglycemic episodes than the chronic complications of the disease [2]. Hypoglycemia can have several effects on the health-related quality of life, like the acute unpleasant symptoms of the episode itself, fear of risk situations and future episodes, anxiety and reluctance to implement intensive insulin regimen to better metabolic control [3].

The quality of life and utilities are different concepts, however, are related to each other. The concept of utility originates in economic theory that deals with decision making and seeks to develop a model to represent how individuals make decisions when faced with uncertain conditions of health, based on their individual preferences [4]. Utility values are obtained requesting individuals to make judgments about their health status, changes in health status or outcomes, and aim to summarize a variety of aspects and concepts related to health and quality of life in a unique measure [5].

From utility data, it is possible to calculate qualityadjusted life years (QALY), which is a very appropriate measure for clinical effectiveness in economic analyzes (cost-utility studies) in certain contexts and diseases, such as diabetes. There are several methodological approaches to estimate utilities, for example, by validated questionnaires (EQ-5D, SF-6D and HUI3) or discrete choice experiments. In the present study, we opted for using EuroQol (EQ-5D) [6] that is a generic and multidimensional tool that has already been successfully applied in many studies and in several countries, including Brazil [7]. These results can be used for economic evaluations of interventions (prevention, management or treatment) that can affect the quality of life of patients with diabetes.

The aim of the study was to evaluate the health-related quality of life and calculate utilities values associated with hypoglycemia in patients with type 1 diabetes mellitus treated in reference centers of the Brazilian Public Health System.

\section{Methods}

A multicenter, cross-sectional and observational study that collected data from patients with T1DM on healthrelated quality of life and hypoglycemia.

The study aimed to recruit 300 patients with T1DM in three public reference centers: Instituto da Criança com Diabetes-ICDRS, Porto Alegre; Instituto de Endocrinologia e Diabetes Luiz Capriglione-IEDE, Rio de Janeiro e Centro Integrado de Diabetes e Hipertensão-CIDH, Fortaleza. Included patients had clinical and laboratory diagnosis of T1DM and more than 18 years. Individuals with any physical or mental conditions that limit the understanding and answering of the questions were excluded.
They were recruited consecutively according to the eligibility criteria and attendance to their medical consultations. Patients answered a structured questionnaire administered by a trained interviewer. The questionnaire included demographic data, information of the disease and its treatment, presence of associated medical conditions, information on intensity and frequency of hypoglycemia and health-related quality of life (EQ-5D and visual analogue scale-VAS).

All subjects were invited to participate and signed a term of consent. The study protocol was submitted to the Research Ethics Committee of each of the executing centers.

\section{Hypoglycemia}

Hypoglycemic events were classified as:

1. Symptomatic hypoglycemia: an event during which typical symptoms of hypoglycemia accompanied or not by a measurement of glucose concentration in plasma $\leq 70 \mathrm{mg} / \mathrm{dl}$.

2. Severe hypoglycemia: an event that requires assistance from another person to actively administer carbohydrate, glucagon, or other resuscitative actions.

3. Nocturnal hypoglycemia: a symptomatic event (documented or not), severe or not, which occurred after 10 p.m. until dawn.

The frequency of hypoglycemic events was defined as daily, weekly, monthly or at least one episode in the last 3 months. High frequency was considered those individuals with daily or weekly episodes, and low frequency for the others.

\section{Health-related quality of life and utility values}

The EQ-5D is a generic instrument that assesses the quality of life through 5 domains: mobility, self-care, usual activities, pain/discomfort and anxiety/depression. For each item, there is gradation 1, 2 and 3 (no problem, some problems and extreme problems), respectively). Combining the results of these five scores produces 243 different health states.

The utility values vary, usually from 0 (equivalent to death) to 1 (equivalent to perfect health), while negative values (worse than death) can be obtained. The different possible health states are judged from this score from 0 to 1 , that is from a conceptual point of view, are valued by reference to death states and perfect health.

For each health state generated by EQ-5D, a value of utility was assigned according to the valuation parameters of health status obtained from the Brazilian validation study [7]. 


\section{Statistical analysis}

The descriptive analysis of the results is presented in terms of mean or median and standard deviation or variance. We used the Mann-Whitney test for comparisons on VAS and utility values. The Kruskal-Wallis test was used to compare categorical variables.

\section{Results}

We included 221 patients, 107 women and 114 men, mean age $29.8 \pm 11.6$ years, BMI $23.7 \pm 4 \mathrm{~kg} / \mathrm{m}^{2}$ and disease duration of $14.2 \pm 9.1$ years.

All patients were using rapid and basal insulins. The basal insulin mean dose was $39.5 \pm 15.2$ and $17.8 \pm 10.4 \mathrm{U} /$ day for rapid insulin. Most of the population (74.8\%) used NPH insulin as basal insulin, with 55 patients $(25.5 \%)$ in use of long-action analogs (47 patients with insulin Glargine and 8 with Detemir). The rapidacting analogs were used by $63.4 \%$ of the population (18 Aspart, 119 Lispro and 2 Glulisine ), the others used regular insulin (Table 1). There were two patients with incomplete data on treatment.

Most patients $(\mathrm{n}=179,80.9 \%)$ reported that they followed the treatment recommendations partially, 21 patients $(9.5 \%)$ followed all the treatment recommendations, while 17 patients did not follow any guidance (7.69\%). Carbohydrate counting was performed by 83 patients (37.8\%).

Regarding chronic complications of diabetes and associated medical conditions, $19.4 \%$ reported having nephropathy, $21.7 \%$ retinopathy, $21.8 \%$ neuropathy, $13.6 \%$ were hypertensive, $0.9 \%$ reported coronary heart disease and $1.8 \%$ reported a previous episode of stroke.

The frequency and severity of hypoglycemia episodes are displayed in Table 2.

The median value of the visual analogue scale (VAS) was $70[60-85]$ and the median utility value was 0.801 [0.756-1.000]. Tables 3 and 4 show the VSA and utility values according to the categories and frequency of hypoglycemia. Figures 1 and 2 demonstrate the utility and VAS according to the frequency of severe hypoglycemia.

\section{Table 1 Treatment according to insulin types}

\begin{tabular}{lclll}
\hline Basal insulins & & & \multicolumn{2}{l}{ Rapid insulins } \\
\cline { 5 - 5 } $\mathbf{N = 2 1 9}$ & & & $\mathbf{N}=\mathbf{2 1 9}$ \\
\hline $\mathrm{NPH}$ & $164(74.48 \%)$ & & Regular & $80(36.5 \%)$ \\
Glargine & $47(21.8 \%)$ & & Aspart & $18(8.2 \%)$ \\
Detemir & $8(3.7 \%)$ & & Lispro & $119(54.3 \%)$ \\
& & & Glulisina & $2(0.9 \%)$ \\
\hline
\end{tabular}

Table 2 Frequency and severity of hypoglycemia episodes

\begin{tabular}{|c|c|c|c|}
\hline Frequency (\%) & $\begin{array}{l}\text { Hypoglycemia }^{a} \\
N=214(96.8 \%)\end{array}$ & $\begin{array}{l}\text { Nocturnal } \\
N=150 \\
(68.1 \%)\end{array}$ & $\begin{array}{l}\text { Severe } \\
N=77(34.8 \%)\end{array}$ \\
\hline Daily & 27 (12.6\%) & $5(3.3 \%)$ & $2(2.6 \%)$ \\
\hline Weekly & 104 (48.6\%) & $53(35.3 \%)$ & $18(23.4 \%)$ \\
\hline Monthly & 67 (31.3\%) & $56(37.3 \%)$ & 19 (24.7\%) \\
\hline $\begin{array}{l}\text { Once (last } \\
3 \text { months) }\end{array}$ & $16(7.5 \%)$ & $36(24 \%)$ & $38(49.3 \%)$ \\
\hline
\end{tabular}

a Any symptomatic hypoglycemia excluding nocturnal or severe episode

\section{Discussion}

The present study aimed to collect data from a group of patients with T1DM treated in the Brazilian Public Health System (SUS) for generating information on health-related quality of life and utility values associated to hypoglycemia. The study population was derived from reference centers for the treatment of T1DM in the South, Southeast and Northeast regions of Brazil. The treatment with basal-bolus regimen was done by all patients, with $25.5 \%$ using slow-acting insulin analogs and $63.4 \%$ using rapid-acting analogs. Approximately $20 \%$ already had chronic complications of diabetes and only $9.5 \%$ followed all the treatment recommendations correctly.

As expected, most of the population reported episodes of hypoglycemia in the last 3 months, with 68 and $34 \%$ reporting nocturnal and severe hypoglycemia, respectively. Of the patients who had severe hypoglycemia, requiring third-party help for their recovery, 26\% reported high frequency of episodes.

We observed the perception of diabetic patients in relation to their health by Visual Analogue Scale assessment. The result was slightly lower than that found in the Brazilian multicenter study of 3.005 T1DM patients (70 vs 72.5) [8] and much lower than the national average obtained in the sample of 9.148 healthy subjects of similar age (70 vs 82.1) [7]. The comparison of VAS and utility values between groups with and without hypoglycemia did not showed significant difference, but the patients with severe hypoglycemia and those with high frequency of episodes (daily or weekly) had lower values than patients who did not report these episodes. These results are consistent with previous studies that have shown that hypoglycemia negatively influences quality of life in type $2[9,10]$ and type 1 diabetic patients [11].

The evaluation of health-related quality of life has been increasingly recognized as an important area of scientific knowledge, as it takes into account various aspects of the patient's life, physical and mental well-being, and 
Table 3 Visual analogue scale and utility values according to the categories of hypoglycemia

\begin{tabular}{|c|c|c|c|c|c|c|c|c|}
\hline \multirow[t]{2}{*}{ Categories } & \multicolumn{4}{|c|}{ Visual analogue scale } & \multicolumn{4}{|c|}{ Utility } \\
\hline & Q1 & Median & Q3 & $p$ value & Q1 & Median & Q3 & $p$ value \\
\hline \multicolumn{9}{|c|}{ Hypoglycemia } \\
\hline No & 50 & 70 & 85 & & 0.787 & 0.801 & 1.000 & \\
\hline Yes & 60 & 70 & 85 & 0.39 & 0.756 & 0.801 & 1.000 & 0.76 \\
\hline \multicolumn{9}{|c|}{ Nocturnal hypoglycemia } \\
\hline No & 60 & 75 & 85 & & 0.787 & 0.801 & 1.000 & \\
\hline Yes & 60 & 70 & 85 & 0.27 & 0.756 & 0.801 & 1.000 & 0.9 \\
\hline \multicolumn{9}{|c|}{ Severe hypoglycemia } \\
\hline No & 61 & 80 & 90 & & 0.787 & 0.801 & 1.000 & \\
\hline Yes & 60 & 70 & 80 & 0.006 & 0.737 & 0.801 & 1.000 & 0.14 \\
\hline
\end{tabular}

Table 4 Visual analogue scale and utility values according to the frequency of nocturnal and severe hypoglycemia

\begin{tabular}{|c|c|c|c|c|c|c|c|c|}
\hline \multirow[t]{2}{*}{ Categories } & \multicolumn{4}{|c|}{ Visual analogue scale } & \multicolumn{4}{|l|}{ Utility } \\
\hline & Q1 & Median & Q3 & $p$ value & Q1 & Median & Q3 & $p$ value \\
\hline \multicolumn{9}{|c|}{ Nocturnal hypoglycemia } \\
\hline Absent & 60 & 75 & 85 & & 0.756 & 0.801 & 1.000 & \\
\hline Low $^{a}$ & 60 & 70 & 85 & & 0.787 & 0.801 & 1.000 & \\
\hline $\mathrm{High}^{\mathrm{b}}$ & 60 & 70 & 81.25 & $0.40^{\S}$ & 0.737 & 0.801 & 1.000 & $0.74^{\S}$ \\
\hline \multicolumn{9}{|c|}{ Severe hypoglycemia } \\
\hline Absent & 61 & 80 & 90 & & 0.787 & 0.801 & 1.000 & \\
\hline Low $^{a}$ & 60 & 70 & 80 & & 0.756 & 0.801 & 1.000 & \\
\hline $\mathrm{High}^{\mathrm{b}}$ & 50 & 62.5 & 72.25 & $0.007^{\S}$ & 0.728 & 0.737 & 1.000 & $0.02^{\S}$ \\
\hline
\end{tabular}

$\S$ Between low and high frequency of hypoglycemia

a Low frequency: one monthly episode or one in the last 3 months

b High frequency: daily or weekly episodes

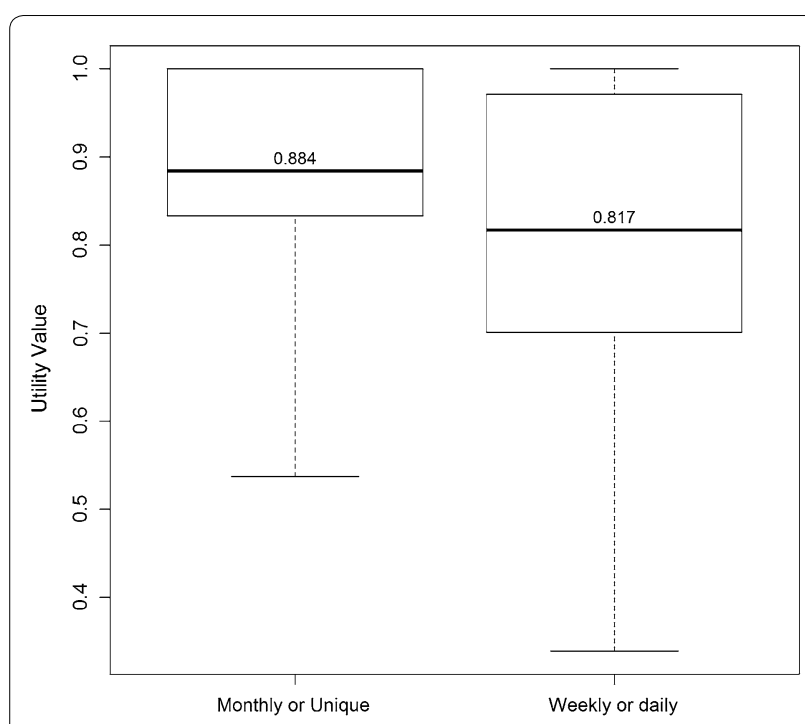

Fig. 1 Utility values in patients with severe hypoglycemia (frequent or rare)

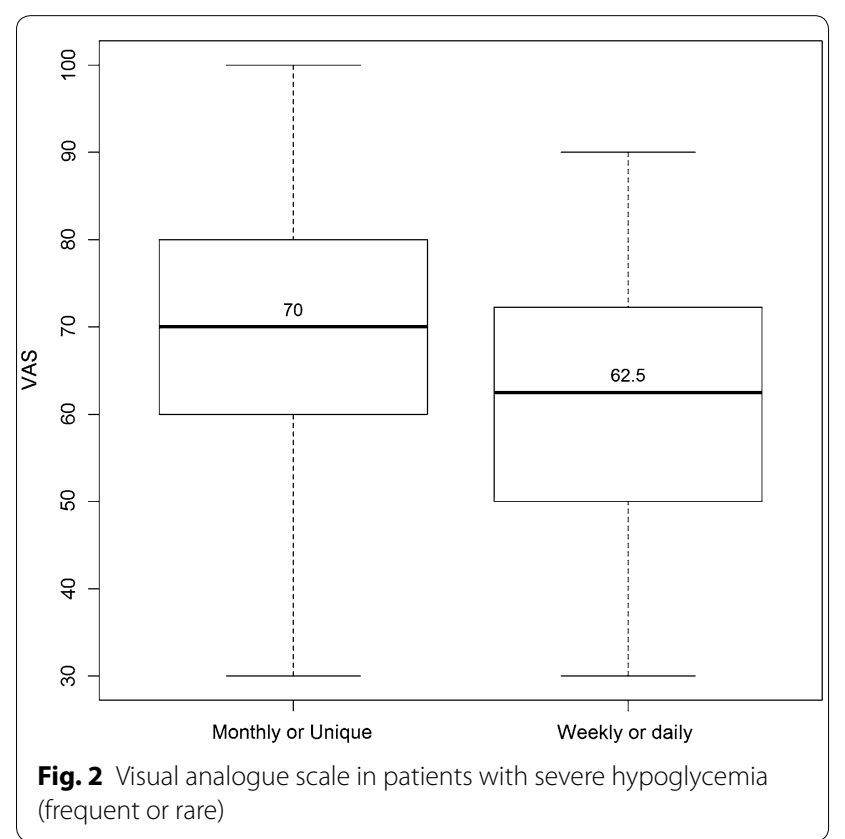


satisfaction with treatments. Several assessment tools have been proposed to evaluate specifically the diabetic population, but many of them have not yet been validated in Brazil [12]. A limitation of this study was the use of a generic instrument for quality of life assessment, that although often used and previously validated in Brazil, which allows comparison with other populations, is not specific to diabetes and therefore has limitations in reaching some aspects of T1DM patients' lives.

There are now a substantial number of studies reporting utility scores for people with diabetes and for common complications associated with the disease [13]. Health technology assessment agencies around the world have consistently adopted the inclusion of utilities (or disutilities) associated with health interventions in economic analysis of new technologies. The premise is that possible effects of treatments can add utility decrements or increments and, therefore, should be considered as a parameter for evaluation beyond the efficacy.

Based on the data from the National Euroqol validation study [7], it was possible to obtain, for the first time utility values specifically for the Brazilian population with T1DM and related to hypoglycemia. Although the values were not different between diabetic patients with and without hypoglycemia, possibly by the small sample size, it was observed that patients who had a high frequency of severe hypoglycemias reported a worse perception of their health status. It must be taken into consideration in treatment decisions by prescribers and health policy makers, especially when thinking of populations of children.

\section{Conclusion}

This study shows the high frequency of hypoglycemia in a sample of T1DM patients treated in three reference centers of the Brazilian public health system and the impact of severe episodes on health-related quality of life. Utility values were generated and can be used in economic analysis for treatments that could decrease hypoglycemia and consequently improve quality of life.

\section{Authors' contributions \\ $L B$ and BRT developed the initial protocol of the study, organized and ana- lyzed data; LB wrote the manuscript and was the responsible for submitting the manuscript; RK, BD and ACF were the coordinators of the centers; DM, DAM, MP, SL, CFSF, ADM collected data of the patients. All authors read and approved the final manuscript.}

\footnotetext{
Author details

${ }^{1}$ Universidade do Estado do Rio de Janeiro-UERJ, Rio de Janeiro, Brazil. ${ }^{2}$ Instituto de Diabetes e Endocrinologia Luiz Capriglione-IEDE, Rio de Janeiro, Brazil. ${ }^{3}$ Instituto da Criança com Diabetes do Rio Grande do Sul-ICDRS, Rio Grande do Sul, Brazil. ${ }^{4}$ Centro de Diabetes e Hipertensão de Fortaleza-CIDH, Fortaleza, Brazil. ${ }^{5}$ Instituto Nacional de Cardiologia-INC, Rio de Janeiro, Brazil. ${ }^{6}$ Visconde de Pirajá 547/501 Ipanema, Rio de Janeiro 22410-003, Brazil.
}

\section{Acknowledgements}

The authors thank the professionals of the centers that help in data collection and all participating patients.

\section{Competing interests}

The authors declare that they have no competing interests.

\section{Availability of data and supporting materials}

Please contact author for data requests.

\section{Ethics}

The study protocol was submitted to the Research Ethics Committee of each of the executing centers. This research was conducted in accordance with the ethical principles established by Resolution 196/96 of the National Health Council and other applicable regulatory requirements.

\section{Funding}

This study was sponsered by FINEP (Financiadora de Estudos e Projetos do Ministério da Ciência de Tecnologia).

Received: 18 August 2016 Accepted: 13 January 2017

Published online: 28 January 2017

\section{References}

1. Seaquist ER, Anderson J, Childs B et al. Hypoglycemia and diabetes: a report of a workgroup of the American Diabetes Association and the endocrine society. http://care.diabetesjournals.org/content/ early/2013/04/12/dc12-2480.full.pdf.

2. Shi L, Shao H, Zhao Y, Thomas NA. Is hypoglycemia fear independently associated with health-related quality of life? Health Qual Life Outcomes. 2014;30(12):167. doi:10.1186/s12955-014-0167-3.

3. Davis RE, Morrissey M, Peters JR, et al. Impact of hypoglycaemia on quality of life and productivity in type 1 and type 2 diabetes. Curr Med Res Opin. 2005;21:1477-83.

4. Campolina AG, Ciconelli RM. Qualidade de vida e medidas de utilidade: parâmetros clínicos para as tomadas de decisão em saúde. Revista Panamericana de Saúde Pública. 2006;19(2):128-36.

5. Drummond MF, Schulpher M. Methods for the economic evaluation of health care programmes. 3rd ed. Oxford: Oxford University Press; 2005.

6. EuroQol (EQ-5D). http://www.euroqol.org/.

7. Santos M, Cintra MACT, Monteiro AL et al. Brazilian valuation of EQ5D-3L health states: results from a saturation study. Med Dec Making. 2016;36(2):253-63. doi:10.1177/0272989X15613521

8. Braga de Souza ACC, Felicio JS, Koury CC, et al. Health-related quality of life in people with type 1 diabetes mellitus: data from the Brazilian Type 1 Diabetes Study Group. Health Q Life Outcomes. 2015;13:204.

9. Marrett E, Radican L, Davies MJ, Zhang Q. Assessment of severity and frequency of self-reported hypoglycemia on quality of life in patients with type 2 diabetes treated with oral antihyperglycemic agents: a survey study. BMC Res Notes. 2011;21(4):251. doi:10.1186/1756-0500-4-251.

10. Flores NM, Gupta S, Goren A, et al. Recent hypoglycemia episodes are associated with poorer quality of life, healthcare resource use, and work impairment among patients with type II diabetes in Brazil. Value Health. 2015;18(7):A865. doi:10.1016/j.jval.2015.09.515.

11. Harris S, Mamdani M, Galbo-Jørgensen CB. The effect of hypoglycemia on health-related quality of life: Canadian results from a multinational time trade-off survey. Can J Diabetes. 2014;38(1):45-52. doi:10.1016/j. jcjd.2013.09.001.

12. Aguiar CCT, Vieira APGF, Carvalho AF, Montenegro-Junior RM. Instrumentos de avaliação de qualidade de vida relacionada à saúde no diabetes melito. Arq Bras Endocrinol Metab. 2008;52(6):931. doi:10.1590/ S0004-27302008000600004.

13. Lung TWC, Hayes AJ, Hayen A. A meta-analysis of health state valuations for people with diabetes: explaining the variation across methods and implications for economic evaluation. Qual Life Res. 2011;20:1669-78. doi:10.1007/s11136-011-9902-y. 